\title{
Exodoncia de diente super- numerario, canino en transposición y enucleación de odontoma complejo: Reporte de Caso
}

Perea-Paz M, Aliaga-Del Castillo R, Aliaga-Del Castillo A. Exodoncia de diente supernumerario, canino en transposición y enucleación de odontoma complejo: Reporte de Caso Rev Estomatol Herediana. 2010; 20(2):90-93.

\section{RESUMEN}

Las anomalías dentarias más frecuentes e importantes que afectan a los niños son la presencia de piezas supernumerarias, piezas retenidas y odontomas. Algunos dientes retenidos se pueden encontrar en transposición. Reportamos el caso de una paciente de sexo femenino de 11 años de edad con presencia de diente supernumerario, canino en transposición y odontoma complejo situados en la hemiarcada derecha del maxilar superior. El motivo de consulta fue por la presencia de dientes anteriores sobresalidos que no le permitía realizar un buen sellado labial. Al examen clínico, presentaba gingivitis generalizada asociada a placa bacteriana, secuencia de erupción retardada por permanencia de pieza 53, ausencia clínica de pieza 13 , aumento de volumen en palatino a nivel de piezas 12 y 11 de consistencia dura y coloración blanquecina que a la presión presentaba isquemia. La presencia del odontoma complejo a nivel cervico mesial de la pieza 16 fue un hallazgo radiográfico. El abordaje quirúrgico se realizó en dos fases debido a las zonas donde se encontraban las tres patologías. El diagnóstico y tratamiento multidisciplinario fueron de vital importancia. En este caso, odontopediatría, radiología, ortodoncia y cirugía estuvieron involucrados.

Palabras clave: DIENTE SUPERNUMERARIO / MIGRACIÓN DEL DIENTE / ODONTOMA.

Extraction of supernumerary tooth, transposed canine and complex odontoma enucleation: a case report.

ABSTRACT

The most common and major dental anomalies affecting children include the presence of supernumerary teeth, impacted teeth and odontomas. Some impacted teeth are found in transposition. We report a case of a 11 years old girl with presence of supernumerary tooth, transposed canine and complex odontoma located on the right quadrant of the maxilla. The chief complaint was the presence of protruding front teeth that did not allow her to do a good lip seal. At the clinical examination, the patient presented generalized gingivitis associated to dental plaque, delayed eruption sequence due to permanence of 53 , clinical absence of 13 , increased volume on the palatal area at 12 and 11 with hard consistency and white areasthat had ischemia to pressure. The presence of complex odontoma at the mesial cervical level of 16 was a radiographic finding. The surgical approach was conducted in two phases because the areas where the three pathologies were. The diagnosis and multidisciplinary treatment were of vital importance. In this case, pediatric dentistry, radiology, orthodontics and surgery were involved.

Key words: SUPERNUMERARY TOOTH / TOOTH MIGRATION / ODONTOMA.

\section{Introducción}

La presencia de dientes supernumerarios, erupcionados o no, puede causar varios problemas en la oclusión por impedir o retrasar la erupción normal de los dientes permanentes, o hasta ocasionar una erupción ectópica. También pueden causar reabsorciones dentarias, pericoronitis, síntomas neurológicos como parestesia y dolor, etc. (1-3). Pueden presentarse como dientes únicos, múltiples o asociados a síndromes.

No existe una etiología exacta, (4), sin embargo la teoría más aceptada es la que señala un desorden hereditario multifactorial que conduce a una hiperactividad de la lámina dental. Los estudios histológicos relacionados a la odontogénesis indican que durante la evolución del germen dentario, la lámina dental degenera, dando lugar a la persistencia de remanentes de lámina dental, ya sea como perlas epiteliales o localizadas en el interior de los maxilares. Si estos restos epiteliales son influenciados por factores inductivos se formará un germen dental que resultará en el desarrollo de un diente supernumerario. (5-7)

La presencia de dientes supernumerarios está asociado también a algunos desórdenes del desarrollo, entre ellos la presencia de labio y paladar fisurado (7-10),

\section{Miguel Perea Paz' \\ Rosalinda Aliaga Del Castillo Arón Aliaga Del Castillo ${ }^{3}$}

'Docente del Departamento Académico de Atención Estomatológica del niño y adolescente.

2Residente Estomatología Pediátrica. Facultad de

Estomatología, Universidad Peruana Cayetano

Heredia.

${ }^{3}$ Cirujano Dentista.

\section{Correspondencia}

Rosalinda Aliaga Del Castillo Jr. JJ Pasos 490-401 - Lima 21, Perú Teléfono: 3324318

e-mail: lindaaliaga@gmail.com

Recibido : 8 de abril de 2010

Aceptado : 20 de mayo de 2010 displasia cleidocraneal (7,9,11-13), y el Síndrome de Gardner (7-9,11). Con menor frecuencia los encontramos en los síndromes de Fabry Anderson (14) y Ellis Van Creveld, entre otros $(7,9,11)$.

Se han reportado casos donde la causa es desconocida y no está asociada a síndromes; estos casos son considerados poco comunes (1519)

Se define a la transposición dentaria como el intercambio posicional de 2 dientes adyacentes o desarrollo o erupción de un diente en una posición normalmente ocupada por un diente no adyacente. Puede ir acompañada por otras anomalías dentales como: agenesias, 
alteración en la forma del diente adyacente, rotaciones severas, dilaceración y malformación de otras piezas, malposición de la pieza adyacente $(20,21)$, tumores, quistes, odontomas, fracturas, dientes supernumerarios, entre otras $(21,22)$.

Se dice que la etiología respondería a una aberración del desarrollo $(21,23)$. Se consideran también otros factores etiológicos podemos mencionar: intercambio de gérmenes dentarios en desarrollo, traumas, alteración en curso de erupción, factores genéticos, presencia de dientes primarios retenidos $(20,21)$.

El odontoma complejo es considerado una neoplasia benigna de origen odontogénico, que se caracteriza por tener tejidos dentarios desorganizados (24).

Su etiología se debe a una alteración durante el periodo de inicio y proliferación de la odontogénesis (estadio de brote y casquete), estadios que se caracterizan por la inducción del ectodermo oral por el ectomesénquima $(25,26)$.

Sus características clínicas son similares al odontoma compuesto, salvo que tiende a ser más común en la región de los molares, y el odontoma compuesto es más de la región anterior (incisivos, caninos) $(24,27)$. Radiográficamente se aprecia como una sombra radiopaca bien delimitada. Histológicamente aparece como una masa de tejido dentario con restos de matriz del esmalte, dentina, cemento y tejido pulpar dispuestos en forma desordenada (27).

\section{Reporte de Caso}

Paciente de sexo femenino de 11 años de edad acude a consulta por presentar los dientes anteriores sobresalidos que no le permiten tener un buen sellado labial. No presenta antecedentes patológicos.
Al examen intraoral presenta secuencia de erupción retardada por permanencia de pieza 53, ausencia clínica de pieza 13, aumento de volumen en palatino a nivel de piezas 12 y 11, de consistencia indurada y coloración blanquecina, que a la presión presenta isquemia (Fig. 1). El informe radiográfico evidenció un diente supernumerario entre piezas 12 y 11 , pieza 13 en transposición, y un odontoma a nivel cérvico mesial de pieza 16 (Fig. 2); Según la técnica de Clark, la pieza supernumeraria y la pieza 13 se encontraban ambas con la corona orientada hacia la tabla ósea vestibular (Fig. 3), mientras que el odontoma se encontraba orientado hacia la tabla ósea palatina (Fig. 4).

Luego de la interconsulta con Ortodoncia, quienes recomendaron la exodoncia de la pieza 13 para evitar reabsorción a nivel de piezas 11 y 12 , se procede a planificar el abordaje quirúrgico del diente

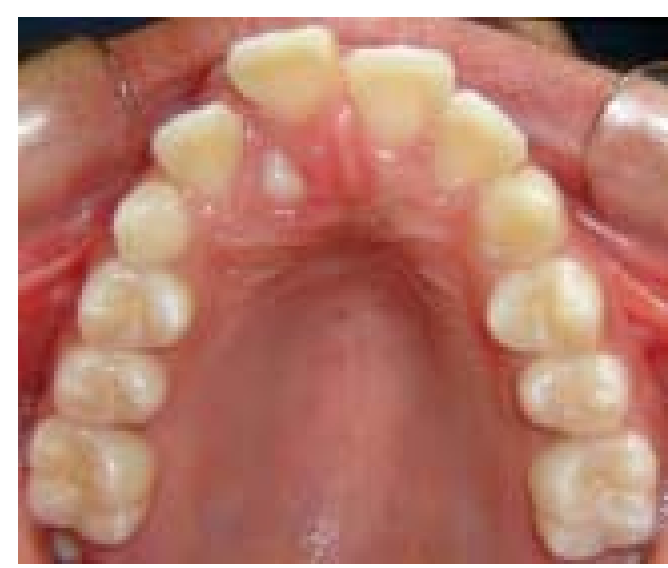

Fig. 1. Maxilar superior.

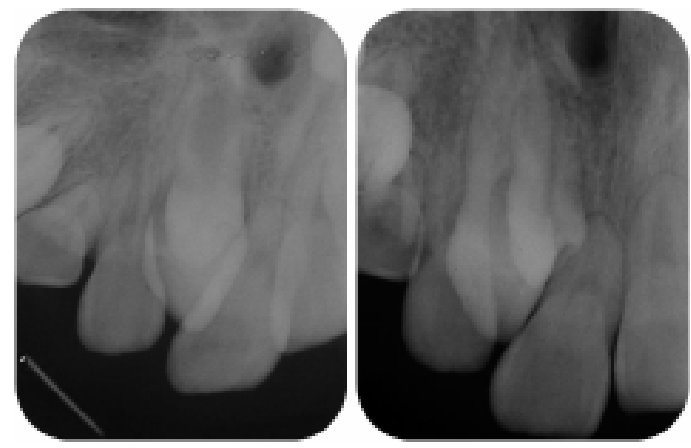

Fig. 3. Radiografía de localización (Tx. Clark).

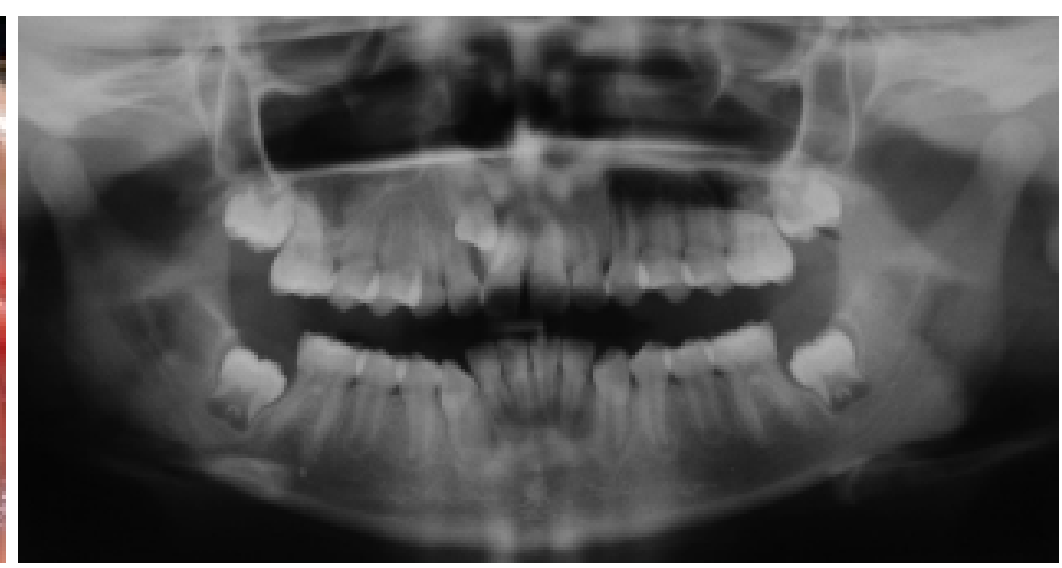

Fig. 2. Radiografía panorámica.
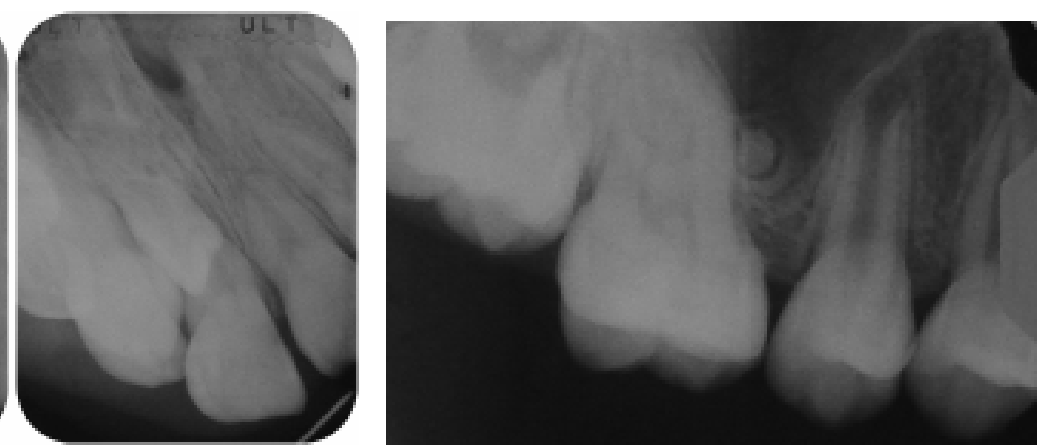

Fig. 4. Odontoma complejo. 
supernumerario, de la pieza 13 ortodoncia.

transposicionada y del odontoma.

El abordaje quirúrgico se realizó en dos fases debido a las zonas donde se presentaban las tres patologías.

La primera intervención (Diciembre 2008) comprendió la exodoncia del canino en transposición y del diente supernumerario, el abordaje se realizó por vestibular para la pieza 13 y por palatino para el supernumerario (Fig. 5 y 6 ).

La segunda intervención (Febrero 2009) comprendió la exodoncia del odontoma, el abordaje se realizó por palatino (Fig. 7 y 8).

Al análisis histopatológico se confirmó que se trataba de un odontoma complejo.

Luego de los controles favorables y después de haberle dado el alta por cirugía y odontopediatría, la paciente continuó su tratamiento en el servicio de

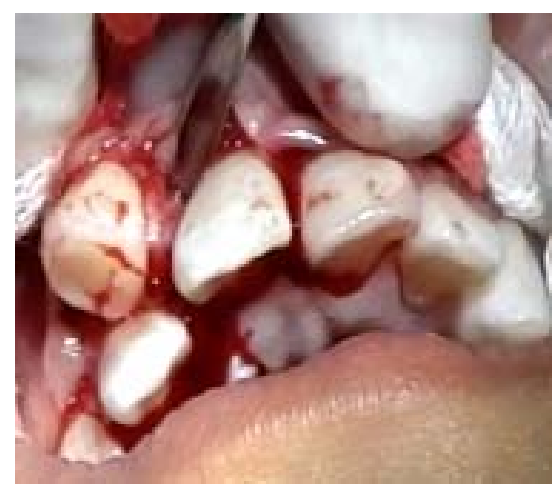

Fig. 5. Fase I.

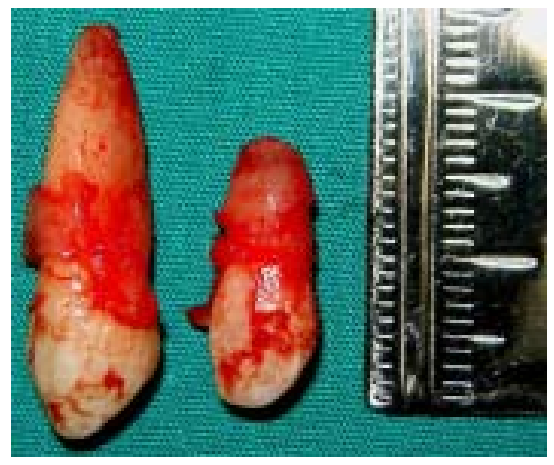

Fig. 6. Canino en transposición y diente supernumerario.

\section{Discusión}

Es raro encontrar en una paciente sin síndrome asociado las tres patologías mencionadas y sobre todo en una misma hemiarcada, hecho que convierte al caso en uno muy singular.

La presencia de dientes supernumerarios, caninos en transposición, y odontomas representan un serio problema y necesariamente deben ser tratados multidisciplinariamente.

La presencia de dientes supernumerarios en pacientes sin síndrome asociado es poco común. Sin embargo Kalra (15), Açikgöz (16), Srivatsan (17), Sasaki (18), Sivapathasundharam (19), han reportado casos similares al de nosotros.

Perez (21) al referirse a la transposición de caninos, señala que

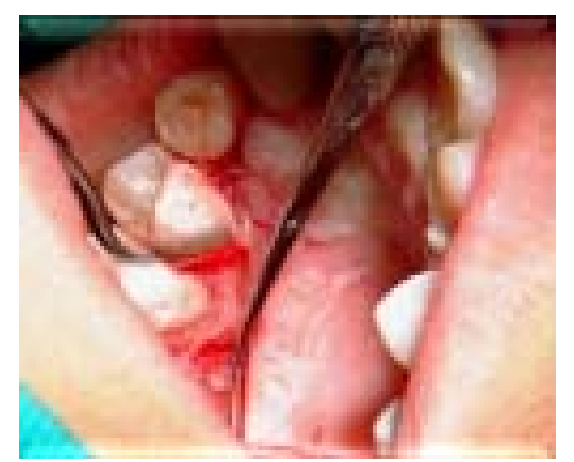

Fig. 7. Fase 2.

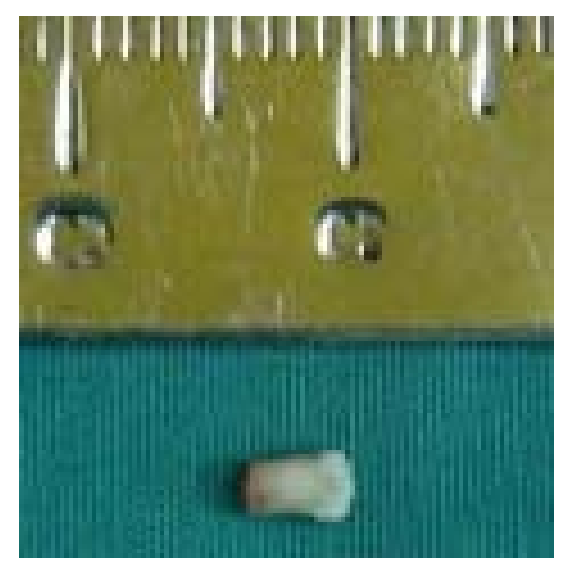

Fig. 8. Odontoma complejo. la consecuencia que adquiere mayor importancia es la reabsorción de las raíces de los incisivos, hecho que cuando ocurre se recomienda la exodoncia de la pieza transposicionada, lo que pasó en nuestro caso.

Patiño (24) y Tomizawa (27), señalan que el odontoma complejo se encuentra comúnmente en la región de molares, lo mismo que ocurrió en nuestro caso, confirmando el diagnóstico con el análisis histopatológico.

\section{Conclusiones}

El tratamiento multidisciplinario es de vital importancia en este tipo de pacientes, en este caso, odontopediatría, radiología, ortodoncia y cirugía estuvieron involucradas.

Es raro encontrar en un mismo paciente sin síndrome asociado, estas tres patologías y sobre todo en un mismo maxilar, lo que le da relevancia necesaria al caso.

El diagnóstico acertado y el tratamiento temprano de estas patologías asumen vital importancia para mejorar el estado estomatológico de la paciente.

\section{Referencias bibliográficas}

1. Sousa Gomes H, Lira Gomes I. Frecuencia y distribución de dientes supernumerarios. Med Oral. 2002: 4(3):84-7.

2. Lozada O. Alteraciones en el número de dientes asociados a maloclusiones [Tesis de Postgrado en Ortodoncia]. Caracas: Universidad Central de Venezuela; 2003.

3. Fernández Montenegro P, Valmaseda Castellón E, Berini Aytés L, Gay Escoda C. Retrospective study of 145 supernumerary teeth. Med Oral Patol Oral Cir Bucal. 2006; 
11(4):E339-44.

4. Canaval C, Perea M, Kanashiro C, Mayo A. Dientes supernumeraries en odontopediatría. Rev Estomatol Herediana 2003; 13(1-2):27-9.

5. Patchett CL, Crawford PJ, Cameron AC, Stephens CD. The management of supernumerary teeth in childhood--a retrospective study of practice in Bristol Dental Hospital, England and Westmead Dental Hospital, Sydney, Australia. Int J Paediatr Dent. 2001; 11(4):259-65.

6. Boj J, Catala M, García-Ballesta C, Mendoza A. Odontopediatría. Barcelona: España; 2000 p. 89106.

7. Rajab LD, Hamdan MA. Supernumerary teeth: review of the literature and a survey of 152 cases. Int J Paediatr Dent. 2002; 12(4):244-54.

8. Salcido-García JF, LedesmaMontes C, Hernández-Flores F, Pérez D, Garcés-Ortíz $M$. Frequency of supernumerary teeth in Mexican population. Med Oral Patol Oral Cir Bucal. 2004; 9(5):407-9; 403-6.

9. Arcuri C, Muzzi F, Romanini G, Cecchetti F, Pujia A, Giancotti A. Supernumerary teeth diagnosis and treatment approach. Six case reports. Minerva Stomatol. 2002; 51(11-12):501-7.

10.Rahman NA, Abdullah N, Samsudin AR, Naing L, Sadiq MA. Dental anomalies and facial profile abnormality of the nonsyndromic cleft lip and palate children in Kelantan. Malays J Med Sci. 2004; 11(2):41-51.

11. Cooper SC, Flaitz CM, Johnston DA, Lee B, Hecht JT. A natural history of cleidocranial dysplasia.
Am J Med Genet. 2001; 104(1):1-6.

12.González López BS, Ortiz Solalinde C, Kubodera Ito T, Lara Carrillo E, Ortiz Solalinde E. Cleido cranial dysplasia: report of a family. J Oral Sci. 2004; 46(4):259-66.

13.Tanaka JL, Ono E, Filho EM, Castilho JC, Moraes LC, Moraes ME. Cleidocranial dysplasia: importance of radiographic images in diagnosis of the condition. J Oral Sci. 2006; 48(3):161-6.

14. Karacay S, Saygun I, Tunca Y, Imirzalioglu N, Guvenc G. Clinical and intraoral findings of a patient with tricho-rhinophalangeal syndrome type I. J Indian Soc Pedod Prev Dent. 2007; 25(1):43-5.

15. Kalra N, Chaudhary S, Sanghi S. Non-syndrome multiple supplemental supernumerary teeth. J Indian Soc Pedod Prev Dent. 2005; 23(1):46-8.

16. Açikgöz A, Açikgöz G, Tunga U, Otan F. Characteristics and prevalence of non-syndrome multiple supernumerary teeth: a retrospective study. Dentomaxillofac Radiol. 2006; 35(3):185-90.

17. Srivatsan P, Aravindha Babu N. Mesiodens with an unusual morphology and multiple impacted supernumerary teeth in a non-syndromic patient. Indian J Dent Res. 2007; 18(3):138-40.

18. Sasaki H, Funao J, Morinaga H, Nakano K, Ooshima T. Multiple supernumerary teeth in the maxillary canine and mandibular premolar regions: a case in the postpermanent dentition. Int $\mathrm{J}$ Paediatr Dent. 2007; 17(4):304-
8.

19. Sivapathasundharam B, Einstein A. Non-syndromic multiple supernumerary teeth: report of a case with 14 supplemental teeth. Indian J Dent Res. 2007; 18(3):144.

20.Ely NJ, Sherriff M, Cobourne MT. Dental transposition as a disorder of genetic origin. Eur $\mathrm{J}$ Orthod. 2006; 28(2):145-51.

21.Pérez FMA, Pérez FP, Fierro MC. Alteraciones en la erupción de caninos permanentes. Int $\mathrm{J}$ Morphol. 2009; 27(1):139-43.

22.Camilleri S, Scerri E. Transmigration of mandibular canines--a review of the literature and a report of five cases. Angle Orthod. 2003; 73(6):753-62.

23. Aydin U, Yilmaz HH, Yildirim D. Incidence of canine impaction and transmigration in a patient population. Dentomaxillofac Radiol. 2004; 33(3):164-9.

24.Patiño C, Berinil Sánchez GE. Odontomas compuestos y complejos: análisis de 47 casos. Arch Odontoestomatol. 1995;11(8):423-30.

25.Boj J. Desarrollo y erupción dentaria. En Odontopediatría. 1ra. Ed. Barcelona, España: Editorial Masson S.A.;2005.

26. Cameron A. Anomalías dentales. En Manual de Odontología Pediátrica. 1ra. Ed. Madrid, España: Editorial Hartcourt Brace;1998.

27. Tomizawa M, Otsuka Y, Noda T. Clinical observations of odontomas in Japanese children: 39 cases including one recurrent case. Int J Paediatr Dent. 2005; 15(1):37-43. 